\title{
Plasmodium asexual growth and sexual development in the haematopoietic niche of the host
}

\author{
Kannan Venugopal@i', Franziska Hentzschel ${ }^{1}$, Gediminas Valkiūnas ${ }^{2}$ and \\ Matthias Marti(i] ${ }^{1 *}$
}

Abstract | Plasmodium spp. parasites are the causative agents of malaria in humans and animals, and they are exceptionally diverse in their morphology and life cycles. They grow and develop in a wide range of host environments, both within blood-feeding mosquitoes, their definitive hosts, and in vertebrates, which are intermediate hosts. This diversity is testament to their exceptional adaptability and poses a major challenge for developing effective strategies to reduce the disease burden and transmission. Following one asexual amplification cycle in the liver, parasites reach high burdens by rounds of asexual replication within red blood cells. A few of these blood-stage parasites make a developmental switch into the sexual stage (or gametocyte), which is essential for transmission. The bone marrow, in particular the haematopoietic niche (in rodents, also the spleen), is a major site of parasite growth and sexual development. This Review focuses on our current understanding of blood-stage parasite development and vascular and tissue sequestration, which is responsible for disease symptoms and complications, and when involving the bone marrow, provides a niche for asexual replication and gametocyte development. Understanding these processes provides an opportunity for novel therapies and interventions.

Gametogenesis

Maturation of male and female gametes.

\section{Meiosis}

Cell division involving chromosome duplication and genetic exchange.
${ }^{1}$ Wellcome Center for Integrative Parasitology, Institute of Infection, Immunity and Inflammation, University of Glasgow, Glasgow, UK.

${ }^{2}$ Nature Research Centre, Institute of Ecology, Vilnius, Lithuania.

*e-mail: matthias.marti@ glasgow.ac.uk
Malaria is one of the major life-threatening infectious diseases in humans and is particularly prevalent in tropical and subtropical low-income regions of the world. According to the WHO, 219 million malaria cases and 435,000 deaths worldwide were reported in 2017, with $>90 \%$ of both cases and deaths in sub-Saharan Africa ${ }^{1}$. The past decade has seen a drastic reduction in malaria cases and deaths worldwide, but this stunning progress has now been halted by widespread emergence of drug resistance in both parasite and vector species. Whereas Plasmodium falciparum dominates in sub-Saharan Africa, Plasmodium vivax is responsible for most cases in many regions of Asia. At least four additional species can infect humans: Plasmodium malariae, Plasmodium knowlesi, Plasmodium ovale curtisi and Plasmodium ovale wallikeri. Many other species of Plasmodium have been reported to cause malaria in vertebrates, including non-human primates (for example, Plasmodium cynomolgi in macaques and Plasmodium reichenowi in chimpanzees), rodents (for example, Plasmodium berghei and Plasmodium yoelli), birds (for example, Plasmodium gallinaceum, Plasmodium relictum and Plasmodium elongatum) and reptiles (for example, Plasmodium mexicanum).
Malaria parasites have a complex life cycle marked by successive rounds of asexual replication across various stages and tissues, both in the intermediate vertebrate host and in the definitive insect host. Sexual stages (gametocytes) are always formed in blood cells in the vertebrate host, whereas gametogenesis and meiosis require transmission to the insect host. In most Plasmodium species, the highest cell numbers are reached during asexual replication in circulating blood cells of the vertebrate host; a small fraction of those asexual parasites differentiate into sexual stages. In the past decade, renewed focus on sexual stages and transmission has unravelled pathways triggering their formation and unique cellular features. Moreover, a series of studies have shown parasite replication and sexual differentiation in the haematopoietic niche of the vertebrate, which adds an unexpected, new feature to the parasite life cycle. In this Review, we discuss the biology of bloodstage malaria parasites, with a particular focus on recent breakthroughs in our understanding of the sexual stage and its development in the haematopoietic niche. We put these findings in an evolutionary context and discuss new avenues for identifying drug targets and strategies to block transmission. 


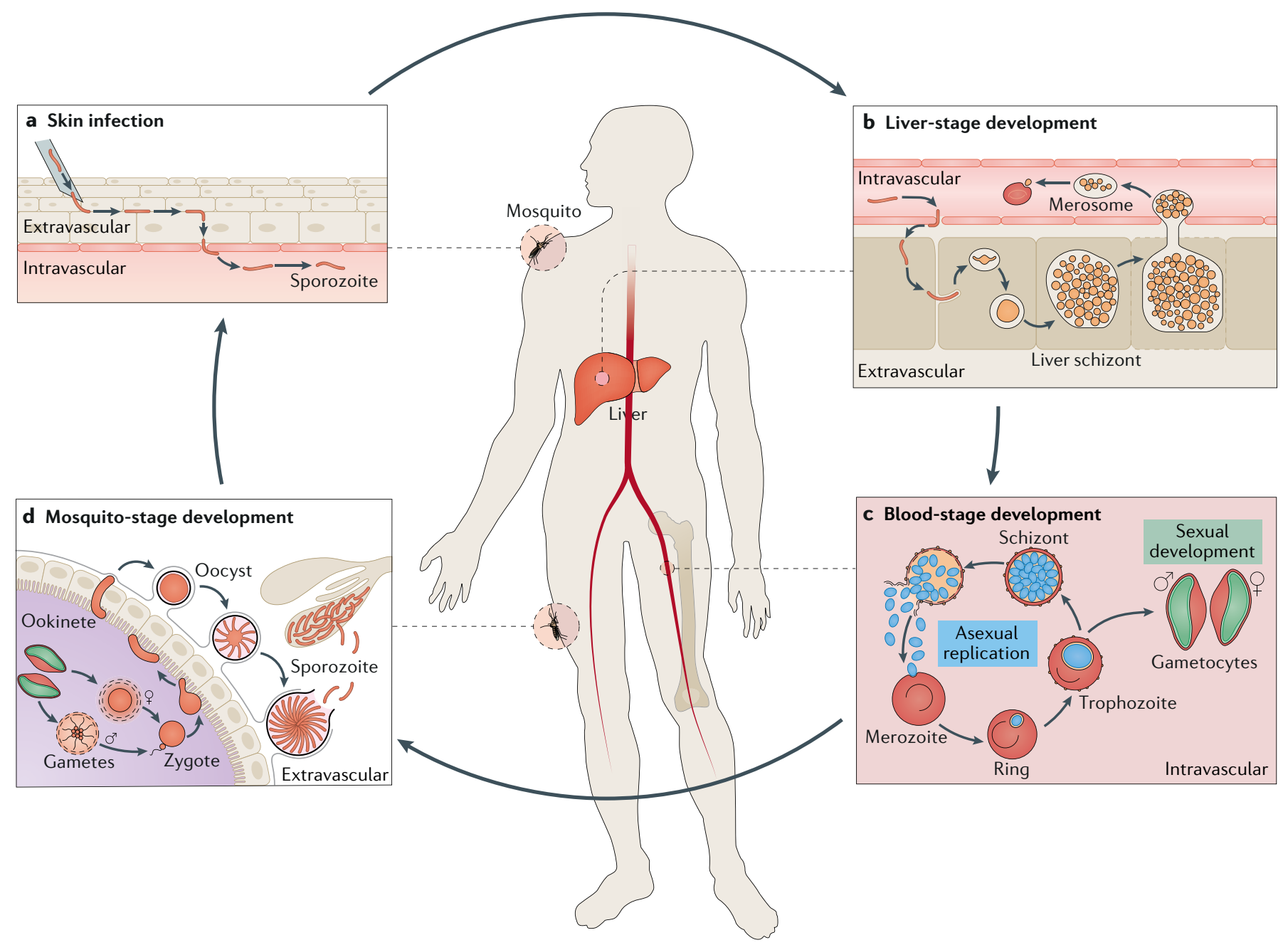

Fig. 1 | Life cycle of Plasmodium falciparum in humans and mosquitoes. a | P. falciparum sporozoites (orange) are injected into the skin during the blood meal of an infected mosquito. They will migrate to and enter a blood capillary. b| Through the bloodstream, the sporozoites reach the liver sinusoids and there they leave the blood circulation to invade a hepatocyte, after multiple transmigration events. In the hepatocyte, they undergo one asexual replication cycle that results in a liver schizont containing thousands of merozoites (yellow). The merozoites enter the bloodstream in membranebound structures termed merosomes. Once released, merozoites infect red blood cells (red) to initiate the intra-erythrocytic parasite cycle. $\mathbf{c}$ In the blood, P. falciparum parasites undergo cycles of asexual replication (blue). After invasion of a red blood cell, they develop from ring stages to trophozoites and then to schizonts. Mature schizonts burst to release merozoites that initiate another replication cycle. A subpopulation of parasites commits to produce male and female sexual progeny or gametocytes (green). $\mathbf{d} \mid$ A female Anopheles mosquito picks up gametocytes while feeding on an infected human. Male and female gametocytes undergo gametogenesis within the midgut of the mosquito. The gametes then fertilize to form a zygote (orange), which further develops into motile ookinetes. Ookinetes cross the midgut epithelium to form an oocyst beneath the basal lamina. In the oocyst, thousands of sporozoites form, which upon bursting of the oocyst wall enter the haemolymph to invade the salivary gland. From there, sporozoites are transmitted to the next human during the subsequent mosquito bite, closing the complex life cycle of the parasite.

\section{Sporozoites}

The only parasite stage that can invade the vertebrate host upon insect bite.

\section{Sinusoids}

Special capillaries lacking a basal lamina and present in the bone marrow, liver, spleen and adrenal glands.

Parasitophorous vacuole A membrane compartment surrounding the parasite and separating it from the host cell.

\section{Plasmodium life cycle}

The features of the malaria parasite life cycle are largely conserved across Plasmodium lineages that infect mammals (FIG. 1). When an infected mosquito takes a blood meal from a vertebrate, it also injects sporozoites into the skin. The motile sporozoite enters the bloodstream, which enables it to reach the liver and thereby escape host immunity or drainage through the lymphatic system ${ }^{2,3}$. Once sporozoites have reached the liver sinusoids, they cross the sinusoidal barrier and enter into hepatocytes ${ }^{2}$, in which they establish a parasitophorous vacuole and differentiate in a first round of asexual replication ${ }^{4}$. Over the course of 2 days to several days (dependent on species), a multinucleated exo-erythrocytic schizont (or meront) containing thousands of daughter merozoites forms. Some parasite species, such as $P$. vivax and $P$. ovale, can then enter a period of latency by forming a non-replicating hypnozoite instead of a schizont. These hypnozoites enable long-term survival of the parasite and can lead to relapses. Upon egress from the hepatocyte, merozoites are clustered in membrane-bound vesicles called merosomes and released back into 


\section{Schizont}

Or meront. A replicative parasite stage in the vertebrate host producing daughter merozoites.

\section{Merozoites}

The only parasite stage that can invade red blood cells

Hypnozoite

A non-replicative dormant

parasite stage in the vertebrate host liver that can reactivate

and lead to relapses.

Reticulocytes

Immature red blood cells developing in the bone marrow before final maturation in the blood circulation.

Microgametes

Male gametes.

Macrogamete

Female gamete

Zygote

A union of male and female gametes where meiosis

takes place.

Ookinete

A motile zygote that forms the oocyst upon crossing the basal lamina of the mosquito midgut.

Oocyst

A replicative stage in the mosquito host producing daughter sporozoites.

Haemolymph Equivalent to blood in arthropods and other invertebrates. the bloodstream via the liver sinusoids ${ }^{5}$. Merozoites invade red blood cells (RBCs), in which a second asexual schizogony takes place. This asexual replication cycle produces up to 32 merozoites over the course of 24-72 h (both parameters vary between species). Through repeated rounds of invasion and growth, the parasite establishes acute and, eventually, chronic infections. Some species, such as $P$. vivax, are restricted to reticulocytes, which make up a small fraction of circulating RBCs, thereby limiting total parasitaemia. Others, such as $P$. falciparum, are not restricted and may infect a high proportion of RBCs leading to high parasite burden, a factor implicated in the capacity of $P$. falciparum to cause severe disease.

The sexual cycle is initiated when a small proportion of asexual parasites commit to produce sexual progeny, that is, gametocytes. Mature gametocytes can circulate in the human blood for several days, which maximizes their chance of transmission to mosquitoes. A few minutes after entering the mosquito midgut, both male and female gametocytes use proteases to exit the RBCs and differentiate into eight microgametes and one macrogamete, respectively ${ }^{6}$, which fuse to produce the zygote. The zygote transforms into a motile ookinete, which crosses the epithelial layer of the midgut wall to form an oocyst. In the oocyst, parasites undergo the third cycle of asexual replication to produce thousands of sporozoites that are released into the haemolymph. Sporozoites that reach the salivary glands of the mosquito attach and invade the gland, where they remain until transmitted to a new vertebrate host through a mosquito bite, to start the cycle again.

Gametocytogenesis. The rate of commitment to sexual development varies widely between species and is determined by a combination of genetic, epigenetic and environmental factors. Initial studies identified chromosomal deletions that lead to the loss of gametocytogenesis of $P$. falciparum during in vitro culture and of $P$. berghei in mice ${ }^{7,8}$. A few years ago, genetic studies in $P$. falciparum and $P$. berghe $i^{9,10}$ identified an essential transcriptional activator of sexual commitment, $a p 2-g$. The ap2-g locus is epigenetically silenced in asexual parasites through the cooperative action of heterochromatin protein 1 (HP1) $)^{11}$ and histone deacetylase 2 (HDA2) ${ }^{12}$. Recently, it was demonstrated that the perinuclear protein gametocyte development 1 (GDV1) ${ }^{13}$ directly interacts with HP1 and derepresses the ap2- $g$ locus ${ }^{14}$, leading to ap2- $g$ transcription and sexual commitment in a subset of schizonts. In P. falciparum, the rate of sexual commitment is sensitive to environmental factors and can be altered depending on in vitro culture conditions ${ }^{15-17}$. Recent work revealed that physiological levels of the human serum phospholipid lysophosphatidylcholine (LysoPC) can repress sexual commitment in vitro ${ }^{17,18}$. LysoPC thereby functions as an environmental signal for nutrient availability in the host as its metabolites are required for membrane biosynthesis and, hence, parasite replication. Whereas $a p 2-g$ is conserved across Plasmodium species, the $g d v 1$ locus and the repressive activity of LysoPC are absent in the subgenus Vinckeia, a rodent malaria lineage of Plasmodium. Besides ap2-g, the earliest detectable transcriptional signature of sexual commitment is an increased expression of a subset of invasion markers ${ }^{17-20}$. A second wave of induced genes encodes many proteins that are exported into the host $\mathrm{RBC}$, the functions of which are discussed below. The process of sexual commitment has been summarized and discussed in detail elsewhere ${ }^{21,22}$.

Depending on the species, gametocyte development takes 1-12 days and results in infectious male and female forms (FIG. 2). At 9-12 days, P. falciparum has the longest (known) gametocyte development, which spans five morphologically distinct phases (stages I-V) ${ }^{23}$. All other studied species from the primate, rodent and avian lineages show subtle morphological changes during gametocyte development and a cycle time between 24 and $60 \mathrm{~h}\left(\mathrm{REF}^{24}{ }^{24}\right.$. During gametocyte development in $P$. falciparum a continuous sheath of microtubules assembles. The microtubules are attached to an array of alveolar sacs beneath the plasma membrane of the parasite, which is called the inner membrane complex (IMC). An IMC can also be found in sporozoites and ookinetes, in which it is required for cellular motility and passage across the sinusoidal and epithelial barrier, respectively. In $P$. falciparum, the establishment of the IMC during early gametocyte development coincides with modifications of the cytoskeleton of the infected RBC (iRBC), including integration of exported parasite antigens, and results in a reversible stiffening of the $\mathrm{iRBC}^{25-27}$. Consequently, stage II-IV gametocytes are more rigid than stage $\mathrm{V}$ gametocytes. Interestingly, the characteristic features of the $P$. falciparum gametocytes (continuous IMC and alterations to RBC cytoskeleton and rigidity) are absent in asexual blood-stage parasites, suggesting fundamental differences in the biology between these two blood stages. It is unclear whether these features are limited to $P$. falciparum gametocytes (and closely related species of the subgenus Laverania) or whether they are more conserved across the Plasmodium lineage.

\section{Vascular sequestration}

P. falciparum can induce cytoadherence of iRBCs to the endothelial cell lining of capillaries and venules in various tissues (FIG. 3), and this process is a major pathogenic mechanism in cerebral and placental malaria ${ }^{28-30}$. Only trophozoites and schizonts cause cytoadherence and sequestration of iRBCs, whereas iRBCs containing ring-stage parasites remain in circulation. Uninfected RBCs and ring-stage iRBCs are biconcave; by contrast, RBCs infected with later asexual stages are spherical, less deformable ${ }^{31}$ and more permeable for small solutes ${ }^{32}$, and their cytoadherence prevents clearance in the spleen. In vitro studies under static and physiological shear flow highlighted the similarities between iRBC cytoadherence and the mechanisms of vascular adherence of leukocytes during an inflammatory immune response after injury ${ }^{33}$. In P. falciparum, the variant surface antigen $P$. falciparum erythrocyte membrane protein 1 (PfEMP1) (3,35 $^{34}$ is the major determinant of cytoadherence. Electron-dense structures called knobs lift PfEMP1 above the dense coat of RBC surface receptors, which facilitates interactions between 


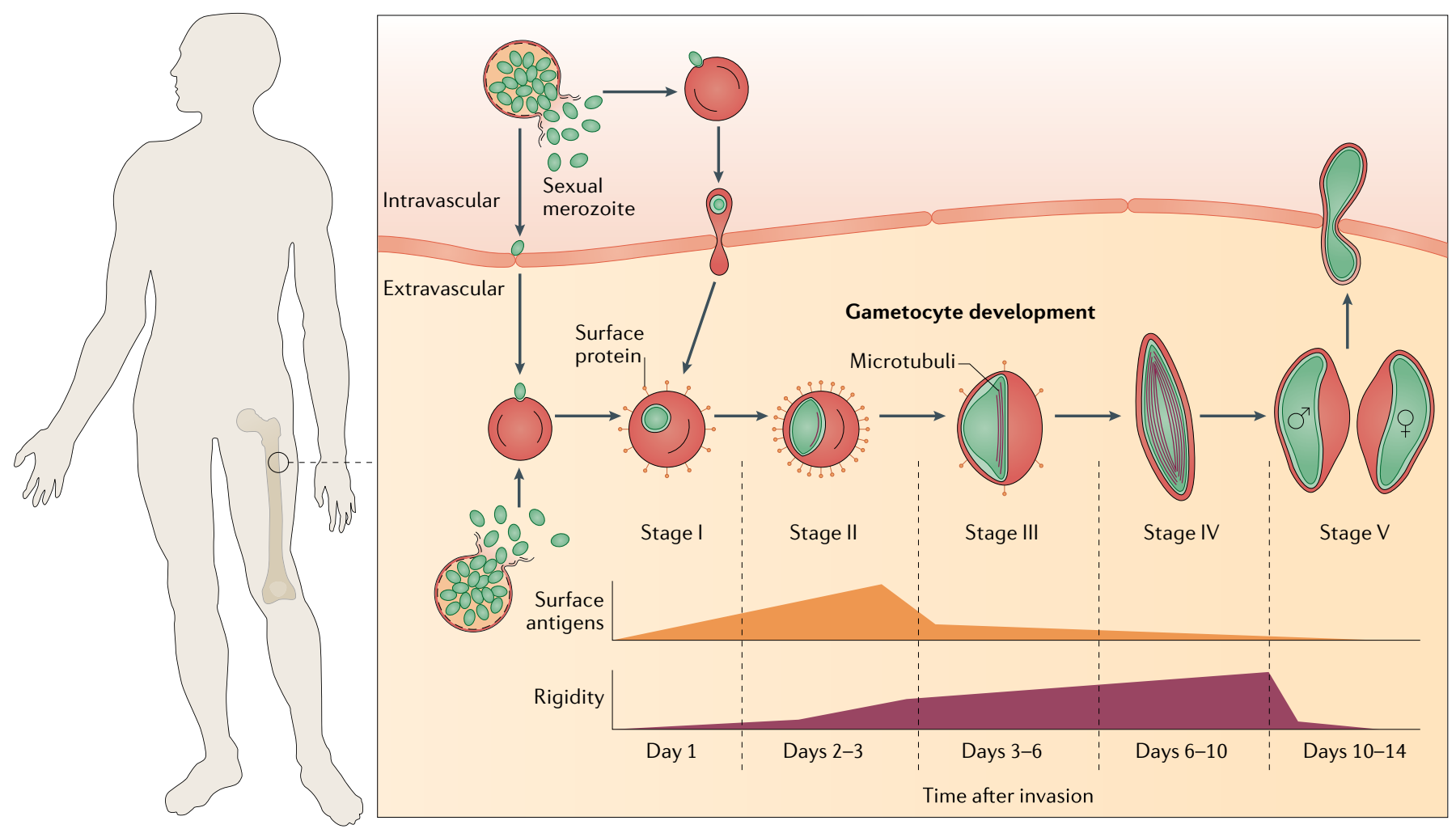

Fig. 2 | Sexual development of Plasmodium falciparum. A subset of schizonts commit to the sexual cycle, producing sexual merozoites. Merozoites and young gametocytes (green) home to the bone marrow, leave the sinusoids and enter the parenchyma. Alternatively, the gametocytes form in the parenchyma from committed schizonts. In the bone marrow parenchyma, gametocytes develop from stage I to stage IV. Remodelling of the membrane of the host red blood cell (red) results in transient deposition of surface antigens (orange) and a reversible increase in cellular rigidity (purple). Restored deformability during maturation to stage $\mathrm{V}$ gametocytes triggers their release back into the bloodstream, where they can be taken up during another mosquito bite. Asexual replication in the bone marrow parenchyma most likely contributes to the accumulation of asexual parasites and sexual commitment in this compartment.

PfEMP1 and endothelial receptors, such as CD36, intercellular adhesion molecule 1 (ICAM1), chondroitin sulfate A (CSA) and endothelial protein $\mathrm{C}$ receptor (EPCR), causing iRBCs to adhere and sequester in the microvasculature and removing them from circulation. Individual PfEMP1 variants have differential binding affinities to host receptors, and the organ-specific distribution or activation of these host receptors determines disease development. Binding of PfEMP1 to EPCR ${ }^{28}$ and ICAM1 (REF. ${ }^{36}$ ) is crucial for brain sequestration (and causal for cerebral malaria), whereas the interactions with $\mathrm{CSA}^{37}$ and $\operatorname{IgM}^{38}$ are required for sequestration in the placenta (and causal for placental malaria). PfEMP1 is the major target of host immunity on the $\mathrm{iRBC}^{39}$ and is under strong selection to maximize its ability both to evade immunity and to bind host receptors. The role of other surface antigens, such as repetitive interspersed families of polypeptides (RIFIN) $)^{40,41}$ and subtelomeric variant open reading frame (STEVOR $)^{41,42}$, in cytoadherence of $P$. falciparum is less clear. However, both variant antigens have been implicated in rosetting, a sequestration mechanism in which iRBCs bind to uninfected RBCs to form clusters that obstruct the microvasculature ${ }^{42,43}$. Parasite-induced modifications of the cytoskeleton and surface of iRBCs, in particular knob structures, increase the likelihood of clearance in the spleen owing to altered biophysical properties of the iRBC. Hence, cytoadherence of iRBCs actively prevents parasites from being in the circulation and, thereby, passing through the spleen. Plasmodium coatneyi is the only parasite in the primate malaria lineage that is known to induce knob-like structures and cytoadherence ${ }^{44}$. The parasite determinants are unknown, however, as both of the major knob components - knob-associated histidine-rich protein (KAHRP) and the major surface ligand, PfEMP1 - are limited to P. falciparum and other members of the Laverania subgenus and, therefore, are absent from P. coatneyi. On the other hand, $P$. vivax increases the deformability of host cells during asexual blood-stage development to facilitate passage through the spleen, and there is no conclusive evidence for parasite accumulation in the brain or placenta and associated pathology in this species ${ }^{45}$.

\section{Sequestration in the bone marrow}

$P$. falciparum gametocytes were first identified by the French physician Alphonse Laveran in blood samples from Algerian soldiers in 1881. Marchiafava and Bignami, two Italian pathologists, found asexual parasite stages in various tissues and observed gametocytes only in the bone marrow and spleen, suggesting that both asexual stages and gametocytes sequester 
Haematopoietic stem cells A cell type that gives rise to all blood cells in the process of haematopoiesis.

\section{Parenchyma}

An extravascular compartment of the bone marrow where

haematopoiesis takes place. during their development ${ }^{46}$. Several case studies identified $P$. falciparum and $P$. vivax gametocytes in the bone marrow and spleen ${ }^{47-50}$, leading to the hypothesis that these organs may represent major sites of gametocyte sequestration. A series of recent studies finally provided quantitative data to confirm these earlier findings (FIG. 4). A histological and quantitative reverse transcription PCR (qRT-PCR) analysis of asexual and immature gametocyte stages in samples from children who died from $P$. falciparum malaria in Malawi showed that the bone marrow is the only organ with substantial gametocyte enrichment, whereas most asexual parasites were found in the spleen, followed by the brain, heart, gut and bone marrow ${ }^{51}$. Analysis of blood samples and bone marrow aspirates from children with P. falciparum malaria and severe anaemia in Mozambique by smears and qRT-PCR showed substantial enrichment of immature gametocytes in the bone marrow compared with the blood ${ }^{52}$. The bone marrow is the major haematopoietic organ in adult mammals, birds and reptiles. It constitutes $\sim 4 \%$ of the total body mass in humans, producing approximately $5 \times 10^{11}$ haematopoietic stem cells per day ${ }^{53-55}$ (all white blood cells, RBCs and platelets). Erythropoiesis occurs in the bone marrow parenchyma, which is connected to the blood circulation through branched sinusoidal vessels. Terminal erythropoiesis occurs in specialized niches, which are called erythroblast islands and consist of a central macrophage surrounded by nucleated RBC precursors ${ }^{56}$. The final nucleated precursor stage is the orthochromatic erythroblast, and once it loses its nucleus, newly formed reticulocytes cross the sinusoidal endothelium to enter the blood circulation. Haematopoiesis outside the bone marrow can occur under both physiological and pathological conditions, in particular in the red pulp of the spleen and in the liver sinusoids. In contrast to humans and all other mammals, the red pulp of the spleen is the major haematopoietic organ in rodents.

In bone marrow samples from children who died from malaria, $50-90 \%$ of all gametocytes were associated with erythroblastic islands. Only young (stage I) gametocytes were found in reticulocytes, suggesting that gametocytes can either form in cells of the erythroblastic island or home to bone marrow as merozoites or in reticulocytes ${ }^{51}$. This finding represented the first quantitative evidence of an extravascular reservoir of bloodstage Plasmodium spp. parasites. Histological analysis in splenectomized non-human primates infected with $P$. vivax confirmed the bone marrow as a primary site of gametocyte enrichment in Plasmodium spp. ${ }^{57}$. Again, most gametocytes were found outside the blood circulation in the bone marrow parenchyma, followed by the liver sinusoids. Moreover, a tissue screen of mice infected with $P$. berghei showed enrichment of immature gametocytes in the parenchyma and sinusoids of the spleen, bone marrow and liver ${ }^{58}$. Finally, P. falciparum infection in immune-deficient mice showed gametocyte accumulation in the bone marrow and spleen ${ }^{59}$. The apparent conservation of this trait across different hosts and three Plasmodium species that have varying gametocyte maturation times (2 days in P. vivax and P. berghei versus 12 days in $P$. falciparum) and morphology (roundish in $P$. vivax and $P$. berghei versus elongated in $P$. falciparum) indicates that the sequestration of immature gametocytes in the bone marrow and secondary haematopoietic organs is a ubiquitous feature of Plasmodium spp. parasites (BOX 1).

Interestingly, the haematopoietic niche is also a reservoir for asexual parasites. In the autopsy case study of

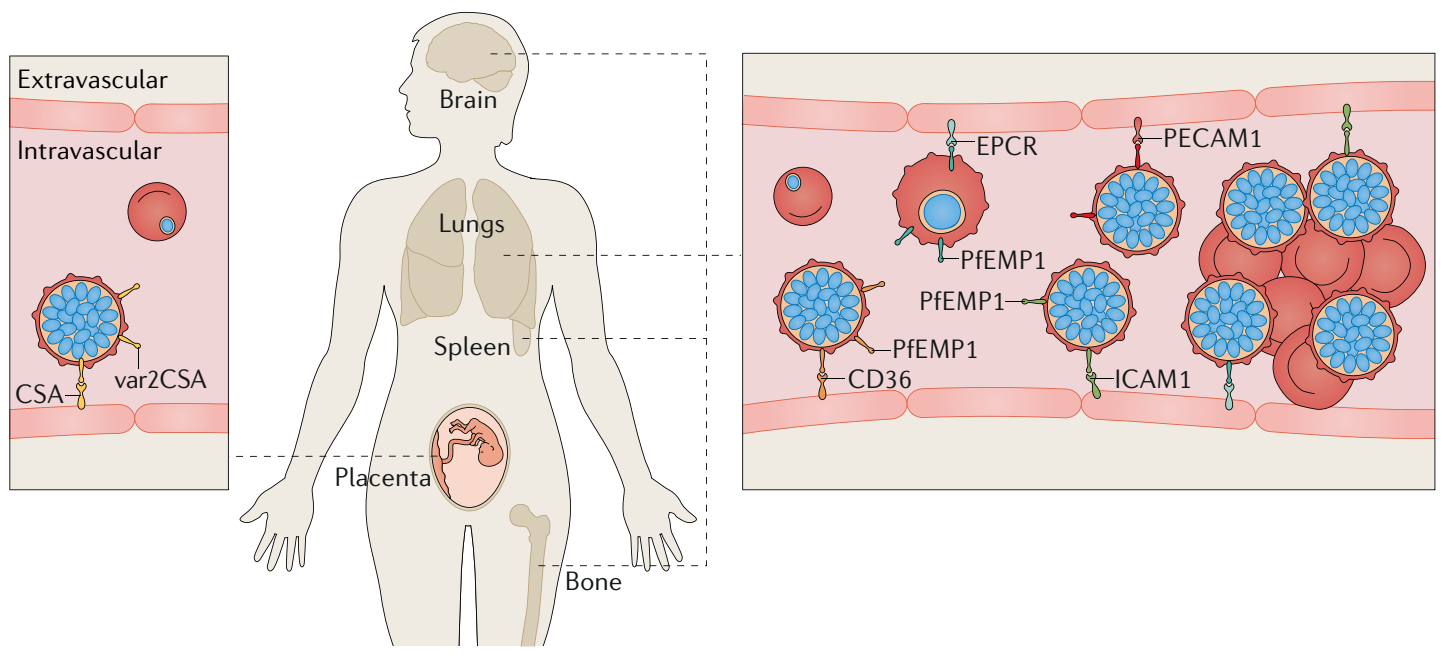

Fig. 3 | Intravascular sequestration of Plasmodium falciparum. Trophozoite and schizont stages of asexual P. falciparum parasites (blue) sequester in the capillaries of several organs, including the brain, lung, spleen and bone marrow. Cytoadherence of infected red blood cells (red) to endothelial cells and to uninfected red blood cells (rosetting) facilitates sequestration. The main parasite ligand is P.falciparum erythrocyte membrane protein 1 (PfEMP1), which is exposed on the surface of infected red blood cells by knob-like structures. The different variants of PfEMP1 interact with diverse endothelial cell receptors, such as endothelial protein C receptor (EPCR), intercellular adhesion molecule 1 (ICAM1), platelet and endothelial cell adhesion molecule 1 (PECAM1) and CD36. In pregnant women, P. falciparum also sequesters in the placenta through the interaction of the PfEMP1 variant var2CSA and the placental receptor chondroitin sulfate $A$ (CSA). Ligand receptor interactions involved in rosetting are not clearly defined, but likely involve repetitive interspersed families of polypeptides (RIFIN) and subtelomeric variant open reading frame (STEVOR) as well as PfEMP1. 


\section{REVIEWS}

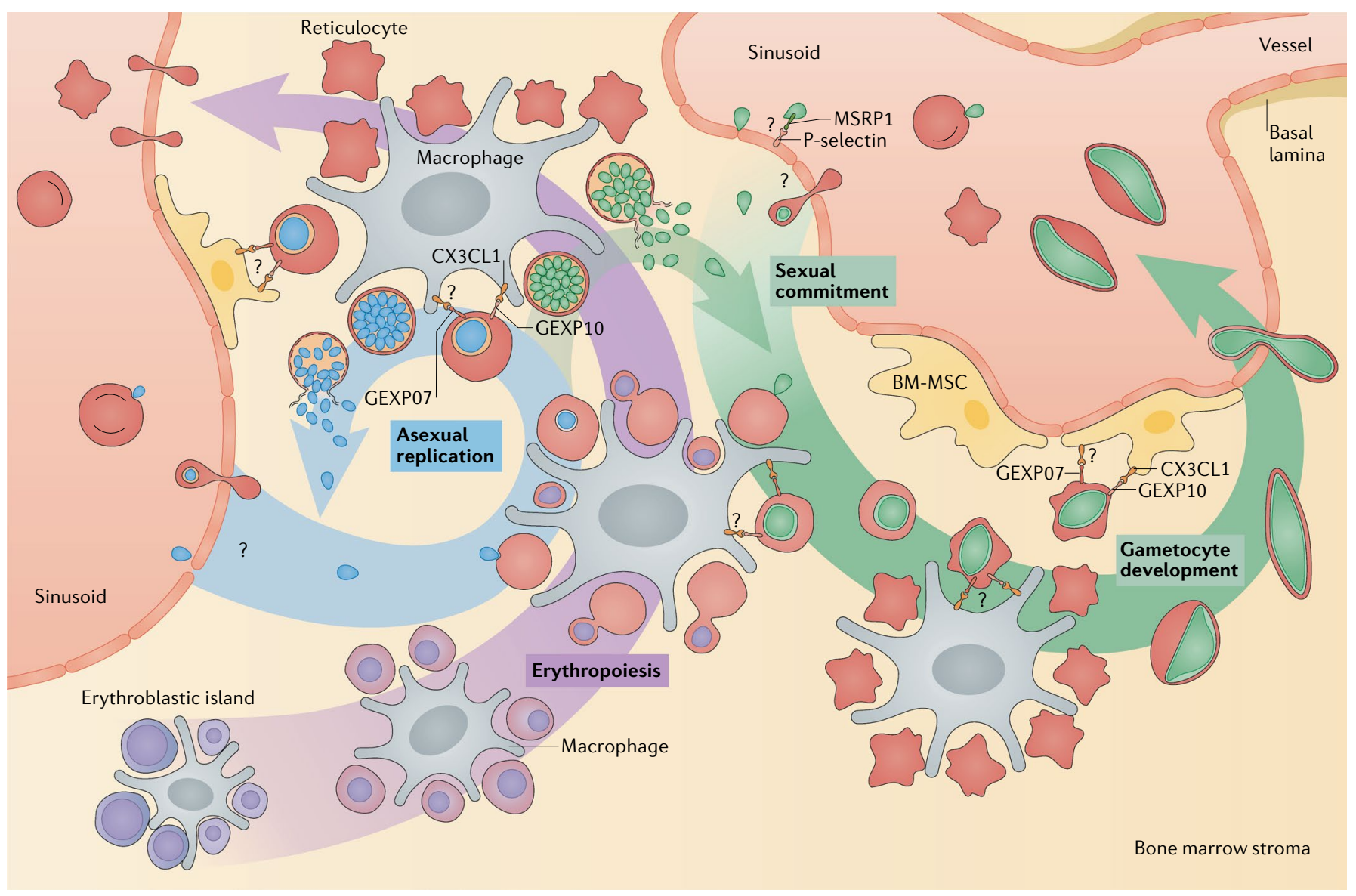

Fig. 4 | Plasmodium falciparum development in the haematopoietic niche of the bone marrow. In the haematopoietic niche of the bone marrow, erythropoiesis occurs in erythroblastic islands, consisting of a central macrophage surrounded by erythroid cells (purple). Coinciding with maturation of the erythroid cells from polychromatic to orthochromatic nucleated red blood cell precursors and then to reticulocytes, erythroblastic islands move closer to the sinusoids. When the reticulocytes have lost their cell nucleus, they are eventually released and enter (either through or between endothelial cells) into the sinusoidal lumen. The asexual parasite cycle in the bone marrow parenchyma is likely established both by influx of asexual merozoites (blue) or ring stages from the sinusoids and by a genuine asexual cycle in the bone marrow. In the parenchyma, asexual parasites may invade and develop in association with erythroblastic islands, or with other cell types. The exported gametocyte-exported parasite proteins (GEXP07 and GEXP10) on the surface of infected red blood cells interact with the host chemokine fractalkine (CX3CL1), which is expressed on different host cells including bone marrow mesenchymal stem cells (BM-MSCs), providing a potential mechanism by which parasites are retained in the bone marrow parenchyma. Gametocytes (green) mature in the bone marrow and are derived either from extravasated sexual merozoites or rings, or from extravascular schizonts that commit to produce sexual progeny in the bone marrow environment. Most gametocytes associate with erythroblastic islands. Stage I and II gametocytes express GEXP07 and GEXP10, which might contribute to bone marrow retention by interacting with other cell types, such as the nursing macrophages and BM-MSCs. Stages III and IV are retained in the bone marrow by their high rigidity, preventing passage through the endothelium. Ultimately, mature stage $V$ gametocytes enter the sinusoids. MSRP1, merozoite surface protein 7-related protein 1.

children with malaria, sequestering $P$. falciparum asexual stages were found in blood vessels, sinusoids and, in particular, the parenchyma of the bone marrow ${ }^{58}$. Moreover, the bone marrow parenchyma and liver sinusoids are the main $P$. vivax reservoir outside the blood circulation in splenectomized non-human primates ${ }^{57}$, whereas the spleen, bone marrow and liver are major reservoirs of asexual $P$. berghei parasites in mice ${ }^{58,60}$. It was generally assumed that $P$. berghei and $P$. vivax parasites do not sequester; however, low levels of $P$. vivax trophozoites and schizonts compared with ring stages in the blood circulation $^{57,61,62}$ and the high frequency of severe anaemia despite low peripheral parasitaemia ${ }^{63}$ have already challenged this assumption. Altogether, these findings demonstrate that the haematopoietic niche of the bone marrow, and other haematopoietic organs, such as the spleen and liver (depending on host species), represent a major reservoir of blood-stage malaria parasites, including asexual stages. This finding is of particular relevance for parasites that prefer, or are restricted to, young RBCs found in the bone marrow, such as $P$. vivax and $P$. berghei.

An extravascular reservoir of asexual parasites may sustain parasite replication and promote gametocyte formation. This hypothesis is supported by the presence of stage I P. falciparum gametocytes in bone marrow 


\section{Box 1 | Evolution of a haematopoietic reservoir in Plasmodium spp. and other haemosporidian parasites}

Life cycles of wildlife Plasmodium spp. and related haemosporidians are diverse and involve various cell types, in particular during exo-erythrocytic development ${ }^{108-110}$. Many haemosporidians have been detected in the haematopoietic niche ${ }^{110-112}$, but the role of this phenotype remains insufficiently understood. For example, all known species of avian Plasmodium spp. belonging to subgenus Huffia multiply mainly in bone marrow stem cells during exo-erythrocytic development, whereas erythrocytic schizonts predominantly form in bone marrow-resident, immature red blood cells ${ }^{110,111}$.

Life cycles of haemosporidians share similarities across the different families and genera, which makes avian Haemoproteus and Plasmodium parasites relevant models for malaria parasites. Notable discoveries based on these models include the identification of sexual stages ${ }^{113}$, exo-erythrocytic development ${ }^{114,115}$ and transmission by mosquitos ${ }^{116}$. Avian malaria parasites were also used for early antimalaria drug screening ${ }^{117}$ and in attempts for in vitro culture ${ }^{118}$. However, there are differences between malaria parasites infecting mammals and those infecting other vertebrates (see figure). All avian and reptile haemosporidians, as well as bat parasites from the genus Polychromophilus, have primary and secondary exo-erythrocytic schizogony, often in diverse cell types ${ }^{109,110}$. Multiple secondary exo-erythrocytic replication cycles often produce a high tissue parasite burden. Some secondary exo-erythrocytic schizonts produce numerous merozoites, which develop into gametocytes that can reach high peripheral parasitaemia. Avian and reptile Plasmodium spp. also show erythrocytic schizogony. Merozoites from erythrocytic and exo-erythrocytic schizonts can initiate secondary exo-erythrocytic schizogony, forming phanerozoites, which often develop in endothelial cells lining the capillaries ${ }^{110,119,120}$. Massive infestation of bone marrow cells by phanerozoites often occurs during infection of birds with Plasmodium elongatum and some other species ${ }^{110,111,121}$. In these infections, phanerozoites are found in haematopoietic tissues, in particular in haematopoietic stem cells. P. elongatum can cause dyserythropoiesis and anaemia due to damage of bone marrow cells ${ }^{111,122}$. The ability of erythrocytic and exo-erythrocytic merozoites to initiate secondary exo-erythrocytic schizogony in avian and reptile parasites opens opportunities for experiments on the tissue stages by inoculation of infected blood, avoiding the use of vectors ${ }^{119}$. Blood schizogony is only reported for Plasmodium spp. of reptiles, birds and mammals ${ }^{110,112}$, and in species of the Garniidae ${ }^{123}$. It is absent in the Leucocytozoidae and Haemoproteidae and in Polychromophilus and has not been reported in Nycteria and Hepatocystis. By contrast, gametocytes are always present in cells of the erythroid lineage, and they often reach high parasitaemia ${ }^{108-110,123-125}$.

Major transitions in life-history traits during haemosporidian evolution are: the emergence of erythrocytic schizogony in the Plasmodiidae species of birds and reptiles, resulting in a second source of gametocyte stages (see figure, part a); and subsequent loss of the secondary exoerythrocytic schizogony in Plasmodiidae species of mammals, resulting in erythrocytic schizogony as the only source of gametocytes (see figure, part b). Despite the marked diversity in morphology and life cycles, haemosporidians share several features in their gametocyte development. First, the gametocyte is the only parasite stage found in cells of the erythroid lineage across all known haemosporidians. Second, gametocytes originate from merozoites developing in schizonts (in the Haemoproteidae and Leucocytozoidae families they form only in exoerythrocytic schizonts; in some members of the Plasmodiidae family (and probably the Garniidae family) they form only in erythrocytic schizonts; and in some members of the Plasmodiidae family (and probably the Garniidae family) they are formed through erythrocytic and exoerythrocytic schizogony ${ }^{110,112}$ ). Interestingly, gametocytes are sexually dimorphic in all known haemosporidians ${ }^{108-110,112}$, in contrast to gametocytes in other apicomplexans.

Importantly, the information about life cycles from wildlife haemosporidians is often based on limited observations ${ }^{108-110}$. For example, the absence of blood schizogony in the bat parasite genera Hepatocystis, Nycteria and Polychromophilus requires further investigation ${ }^{125,126}$, given its presence in closely related species infecting other hosts ${ }^{126,127}$ and the lack of an alternative gametocyte source. Blood schizogony either evolved once and subsequently was lost in the above bat parasites or developed independently several times ${ }^{126,128}$. Altogether, the distribution of life cycles across haemosporidians suggests that establishment of a bone marrow reservoir emerged with blood schizogony, either in parasites infecting birds and/or once they infected mammals (with bats as the most likely origin).

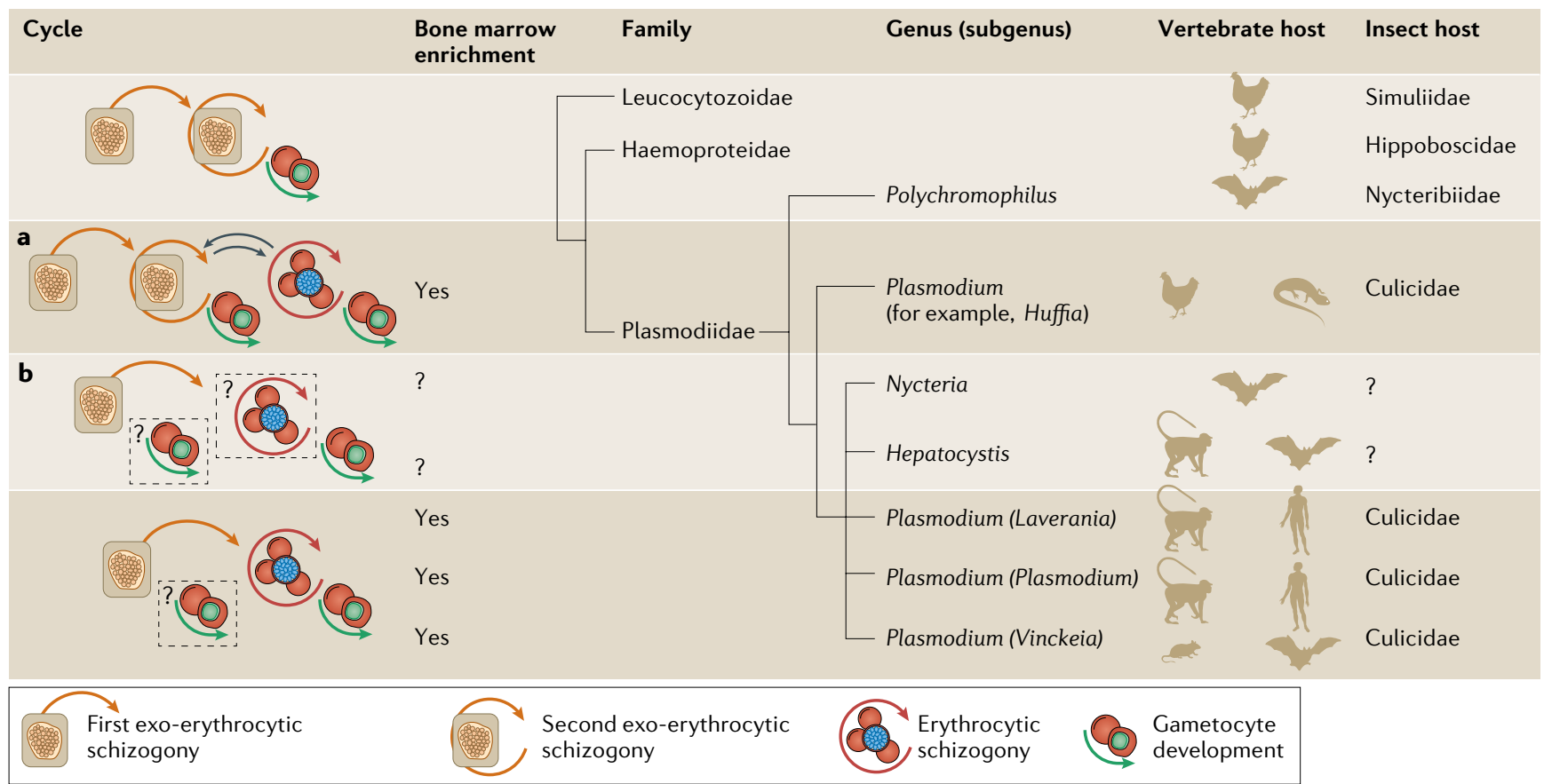


Phanerozoites

Secondary exo-erythrocytic schizonts in avian and reptile malaria parasites

Dyserythropoiesis Defective development of red blood cells, or erythropoiesis. reticulocytes ${ }^{51}$ and the observation that splenic reticulocytes are the major source of gametocytes in a blood-stage $P$. berghei infection ${ }^{60}$. The conditions in the haematopoietic niche may be conducive for gametocyte commitment. Indeed, levels of the major physiological repressor of gametocyte commitment, the host phospholipid LysoPC, are much lower in the bone marrow than in the blood ${ }^{17}$. In addition, $\sim 50 \%$ of all parasites in the bone marrow are gametocytes, both for P. falciparum and $P$. vivax, whereas they make up fewer than $5 \%$ of all parasites in the peripheral blood ${ }^{51,57}$. Alternatively, gametocyte formation may be triggered in the vasculature, possibly through local LysoPC depletion at sites of parasite sequestration, systemically during inflammation ${ }^{17}$ or simply in a stochastic manner. In such a scenario, young gametocyte forms must be detectable in the blood circulation and able to home to the bone marrow before becoming too rigid and cleared by the spleen. Indeed, early gametocyte transcripts including ap2-g are present at detectable levels in patient blood both by qRT-PCR and microarray ${ }^{64-67}$, enabling quantification of the rate of conversion to parasites committed to sexual development in vivo ${ }^{64}$. Importantly, experiments with $P$. berghei also provide indirect evidence of homing and extravasation of a subset of merozoites to the bone marrow parenchyma. In these experiments, a series of endothelial receptors were blocked using specific antibodies prior to infection with $P$. berghei merozoites ${ }^{58}$. Inhibition of either P-selectin or a combination of ICAM1 and vascular cell adhesion molecule 1 (VCAM1) reduced the number of young gametocytes (but not asexual parasites) in early reticulocytes in the bone marrow, suggesting an involvement of these receptors in merozoite extravasation to the bone marrow $^{58}$. Interestingly, human P-selectin has been found in vitro to interact with members of the family of merozoite surface protein 7-related proteins (MSRPs) in $P$. falciparum, $P$. vivax and $P$. berghei ${ }^{68}$. Moreover, singlecell and bulk transcriptome analyses have consistently shown an upregulation of $m s r p 1$ and a second merozoite antigen, dblmsp2, in sexually committed $P$. falciparum schizonts ${ }^{17-19}$. It is therefore tempting to speculate that these two antigens are involved in extravasation and/or host cell invasion of sexual merozoites in the bone marrow. Interestingly, experiments in the rodent model also provided evidence for homing of uninfected and ring-stage iRBCs to the bone marrow, including the extravascular compartment ${ }^{58}$. Altogether, there is evidence for both a genuine sexual commitment cycle in the bone marrow and for intravascular commitment with subsequent homing to this niche. However, more research is needed to understand the molecular mechanisms of parasite entry and exit at the bone marrow interface. Whereas merozoites are invasive stages with the intrinsic ability to invade and possibly migrate across host cells, transmigration of ring-stage iRBCs is more likely driven by the same machinery that facilitates intravasation of reticulocytes. Furthermore, the relative contribution of sexual commitment in the bone marrow versus in the blood to overall levels of gametocyte formation is unknown, but there is likely variation according to host conditions, according to host intrinsic parasite factors and between parasite species.
Host cell modifications required for extravascular sequestration. Asexual $P$. falciparum parasites efficiently bind to the bone marrow endothelium. In fact, panning experiments of iRBCs with endothelial cells from various tissues demonstrated that bone marrow-derived endothelial cells (BMECs) share binding properties with those from the brain ${ }^{69}$. Given the preferred localization of immature gametocytes among erythroblast islands, this parasite stage may interact with RBC precursors, including reticulocytes and/or macrophages, but not with BMECs. Early studies suggested adhesion of immature $P$. falciparum gametocytes to ICAM1 on BMECs ${ }^{70}$. However, further analysis demonstrated only minimal binding of immature gametocytes to BMECs compared with asexual stages ${ }^{71,72}$, arguing against a classical PfEMP1-mediated vascular cytoadherence mechanism. Indeed, both PfEMP1 and KAHRP are epigenetically silenced in gametocytes and these proteins are absent in all gametocyte stages ${ }^{71,73}$. Interestingly, a recent study reported the binding of both asexual stages and immature $P$. falciparum gametocytes to human bone marrow mesenchymal stem cells (MSCs) in a 3D culture system. This binding was trypsin-sensitive, but independent of PfEMP1 and ICAM1, yet the receptor for the interaction could not be identified ${ }^{74}$. In a separate study, no binding to RBC precursor cells was observed ${ }^{75}$. An initial proteomic analysis of immature gametocytes identified a large number of gametocyte-exported parasite proteins (GEXPs) and potential ligands for adhesion ${ }^{76}$. More recently, surface proteomics of immature gametocytes confirmed several of these exported proteins as gametocyte surface antigens, including GEXP07 and GEXP10 (REF. ${ }^{77}$ ). These two antigens have independently been characterized in asexual-stage parasites and were shown to interact with the human chemokine fractalkine $(\mathrm{CX} 3 \mathrm{CL} 1)^{78}$. CX3CL1 is a transmembrane protein expressed in many endothelial cells but also in bone marrow stromal cells, including MSCs and macrophages, and is responsible for retaining monocytes within the bone marrow $^{79}$. As GEXP07 and GEXP10 are CX3CL1 receptor mimics, they may be involved in interactions between asexual and immature gametocytes and cells in the extravascular bone marrow niche, such as MSCs and macrophages. In addition to GEXP07 and GEXP10, most gametocyte antigens are also expressed in asexual parasites, suggesting shared functions in host cell interactions ${ }^{77}$. Given the shared binding phenotype to human bone marrow MSCs, it is most likely that such shared antigens are also involved in iRBC interactions in the extravascular bone marrow niche. Interestingly, the presence of parasite antigens on the gametocyte iRBC surface is limited to stages I and II, after which the antigens are gradually removed by as yet unknown processes ${ }^{77}$, coinciding with the observed loss of binding ${ }^{74}$.

Invasion assays with $P$. falciparum and $P$. berghe have demonstrated that the earliest RBC precursor stage that can be invaded and support parasite growth is the orthochromatic erythroblast ${ }^{51,60,80}$, in the final 48-72 $\mathrm{h}$ of erythropoiesis. Interestingly, the remaining maturation times of invadable RBC stages and of the asexual parasite stage are similar across all known Plasmodium species. Likewise, gametocyte maturation 
of all Plasmodium species so far investigated, except for $P$. falciparum, takes a similar length to the asexual cycle. By contrast, the maturation of $P$. falciparum gametocytes takes 10-14 days, during which they need to avoid premature release of the iRBC into circulation. The benefit of such an unusually long developmental time is not known, except that it is accompanied by an equally unusual level of host cell remodelling. Immature $P$. falciparum gametocytes become increasingly rigid up to stage IV before reverting into a deformable state at stage $\mathrm{V}^{27,81,82}$. The rigidity switch at the onset of stage $\mathrm{V}$ gametocytes is preceded by the loss of surface antigens on the iRBC surface between stage II and III gametocytes $^{77}$, suggesting that the two processes are part of a coordinated remodelling of the host cell. A rigidity differential may serve the same purpose as in reticulocytes $^{83}$, facilitating mechanical retention of the immature gametocytes, whereas only the mature infectious stage $\mathrm{V}$ is sufficiently deformable to enter the blood circulation. The switch to the deformable stage $\mathrm{V}$ appears to be triggered by a drop in the intracellular levels of cAMP, and drugs that increase cAMP levels (for example, the phosphodiesterase inhibitor sildenafil citrate) increase the stiffness of mature gametocytes ${ }^{84}$. In mice, sildenafil citrate increased the number of $P$. berghei gametocytes in the bone marrow and spleen, supporting the notion that the deformability switch is required for a release of mature gametocytes into the circulation ${ }^{58}$. Changes in the parasite cytoskeleton and parasite-induced modifications of the host cell cytoskeleton have been suggested to underlie the deformability switch. P. falciparum gametocytes undergo drastic morphological changes during maturation, building up an extensive cytoskeleton of longitudinal microtubules until stage IV, which give these cells the classical elongated shape. Upon maturation to stage $\mathrm{V}$, this microtubule cytoskeleton is disassembled, likely contributing to the increased deformability of this stage $^{25}$. The variant STEVOR antigen that localizes to the erythrocyte membrane has been implicated in regulating the deformability of gametocytes by interacting with the ankyrin complex, a component of the erythrocyte cytoskeleton $^{81,85}$. Deformability seems to depend on the phosphorylation status of STEVOR, which is regulated by intracellular cAMP levels, and STEVOR dephosphorylation and internalization render mature gametocytes deformable ${ }^{85}$. Yet even STEVOR ${ }^{-}$parasites partially retain rigidity during gametocyte maturation and respond to sildenafil citrate treatment, suggesting that other unknown mechanisms contribute to the deformability switch ${ }^{85}$. Notably, P. berghei and P. vivax gametocytes do not undergo major shape changes or build-up of a microtubule cytoskeleton during maturation. As their maturation time is only 2 days, and thus much shorter than for P. falciparum gametocytes, it is possible that gametocytes in these species do not need a mechanism for continued extravascular retention, but simply mature and enter the blood together with the host reticulocyte. Importantly, there are differences in parasite distribution within the extravascular bone marrow compartment between $P$. falciparum, $P$. vivax and $P$. berghei: whereas autopsy data from human $P$. falciparum infections and necropsies of non-human primates infected with $P$. vivax found most asexual and gametocyte stages in the parenchyma $^{51,58}$, intravital imaging and necropsy data from infected mice revealed a more even distribution between the parenchyma and sinusoids for $P$. berghei $i^{58}$. The physiological relevance and underlying host-pathogen interactions related to these subtle but notable differences remain to be investigated. Intravital imaging has provided direct evidence of mature $P$. berghei gametocyte intravasation and leukocyte-like movement in both the extravascular and intravascular compartments of the bone marrow and spleen ${ }^{58}$. Similar phenotypes have yet to be investigated in $P$. falciparum and $P$. vivax. Mature gametocytes of all species must be ingested by mosquitoes during a blood meal and hence present in the peripheral blood circulation of the dermis. There has been some speculation about a mature gametocyte reservoir in the peripheral microcirculation of the skin $^{86,87}$, but so far there is no experimental evidence for such a phenotype.

Bone marrow infection as a challenge and opportunity for interventions. The unique properties of the bone marrow niche provide both challenges and opportunities for interventions against malaria parasites and other infectious agents (FIG. 5). Currently used artemisinin combination therapies combine the fast-acting and short-lived artemisinin with a long-lasting partner drug, such as mefloquine or piperaquine ${ }^{1}$. These treatment regimens are based on bioavailability and efficacy measurements in the vascular blood compartment, which may be inadequate for parasite clearance in the bone marrow. For example, bone marrow-resident RBC precursors are metabolically more active than mature RBCs, and therefore intracellular pathogens, such as malaria parasites, may be exposed to reduced drug concentrations in these host cells owing to their increased turnover $^{88}$. In addition, drug availability is reduced in the bone marrow owing to limited perfusion ${ }^{89}$. Exposing parasites to sublethal drug concentrations in this compartment mimics current in vitro protocols to select for drugresistant parasites ${ }^{90}$ and therefore may increase the likelihood of emergence and spread of drug resistance. Some antimalarials, including artemisinin, also induce haemolysis and subsequent erythropoiesis in the bone marrow and spleen of infected mice and hence increase infection levels in the haematopoietic niche ${ }^{60}$. On the other hand, the unique metabolic environment of the bone marrow may also provide opportunities to increase the activity of drugs, as shown recently for the prodrug primaquine ${ }^{91}$. In addition, loss of mature gametocyte deformability in P. falciparum ${ }^{84}$ and gametocyte accumulation in the bone marrow and spleen in P. berghei $i^{58}$ upon sildenafil citrate treatment provide a proof-of-concept for a transmission-blocking drug. Systematic efforts using cellular and target-based high-throughput screens are underway to further explore blocking the deformability switch as a drug target ${ }^{92,93}$. Bone marrow infection likely has an effect on the development of immunity, as the environment is naturally immune protected through mechanisms of central tolerance ${ }^{94}$. Parasite infection also induces changes in the haematopoietic compartment ${ }^{95}$ and loss of $\mathrm{B}$ cell populations in the bone marrow ${ }^{96,97}$, 


\section{Recrudescence}

The recurrence of detectable parasitaemia upon clearance to submicroscopic levels. which may be linked to the slow acquisition of protective immunity in malaria. However, parasite sequestration in the bone marrow may also be an opportunity to induce immunity for vaccine development. Identification of shared iRBC surface antigens that are involved in interactions of asexual parasites and gametocytes with the bone marrow could provide the basis for a new bloodstage vaccine that reduces the parasite burden and transmission simultaneously. Indeed, natural human antibodies against such shared antigens correlate with a reduced burden of asexual parasites and mature gametocytes in patients with malaria ${ }^{77}$. Longitudinal studies are required to identify and validate functional antibodies that could serve as a template for next-generation vaccine development. Persistence and recrudescence of a parasite reservoir in the bone marrow and spleen, as described in the rodent model upon drug treatment ${ }^{60}$, also create diagnostic challenges as infections may go undetected. It will be important to identify host and/or
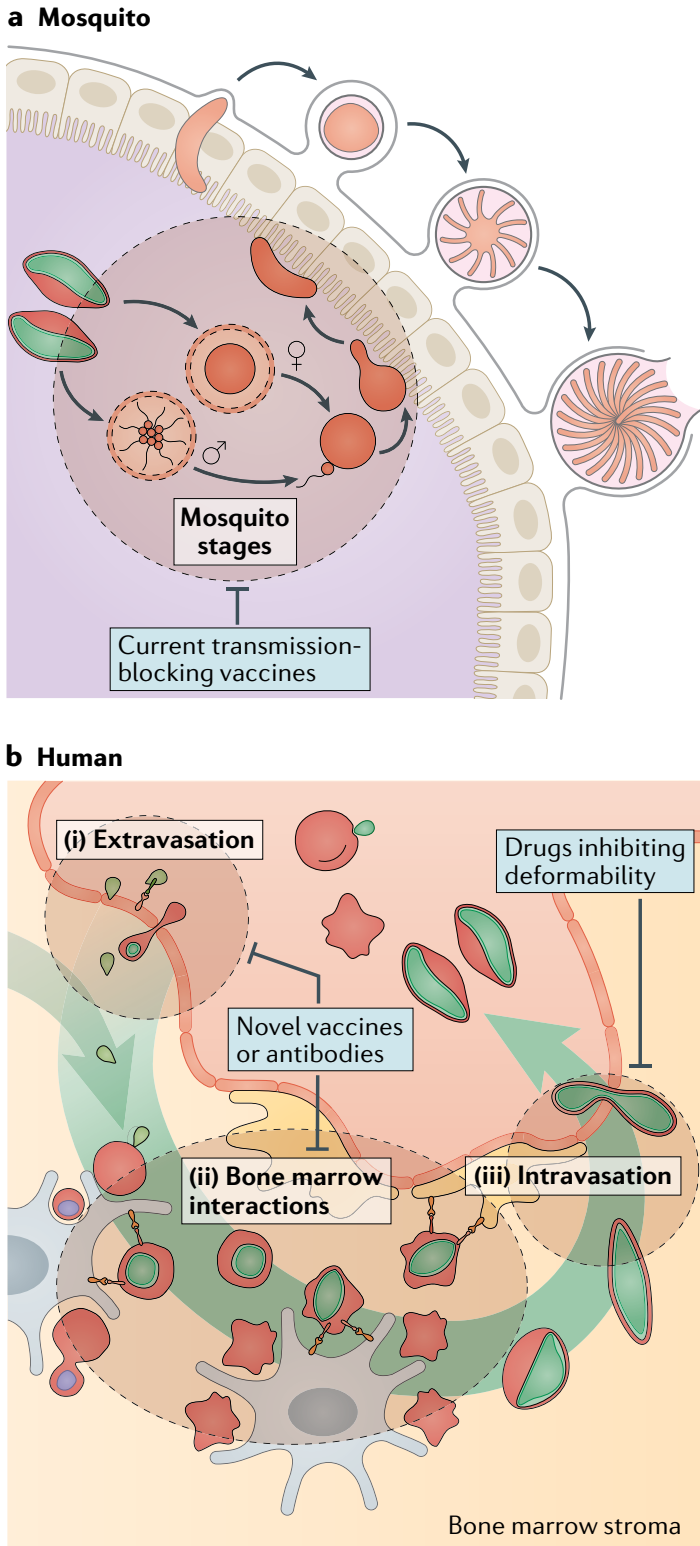

parasite markers for bone marrow infection independently of peripheral parasites to define the true parasite reservoir in the population. An asymptomatic or undetected bone marrow parasite reservoir may be particularly relevant for parasite species that are either reticulocyte-restricted or have latent phases - or both, as is the case for $P$. vivax ${ }^{98}$.

\section{Conclusion and future directions}

The haematopoietic niche is host to various infectious agents due to its nutrient-rich environment, its antiinflammatory and, hence, immune-protected state, and its capacity to produce many of the circulating immune cells and blood cells that harbour pathogens ${ }^{99}$. For example, the causative agent of tuberculosis, Mycobacterium tuberculosis, can enter bone marrow MSCs. The bone marrow may represent a reservoir for latent $M$. tuberculosis infection, as non-replicating yet viable bacteria were successfully isolated from the bone marrow of patients who had undergone antituberculous treatment and been declared disease free ${ }^{100}$. Likewise, splenic sequestration in visceral leishmaniasis has been reported, although exclusively in the context of active disease ${ }^{101}$.

Identification of the bone marrow as a primary site of gametocyte development and a major reservoir for asexual parasites in Plasmodium spp. represents a fundamental shift in our understanding of parasite biology and opens up a new research field in the malaria community. There have also been a series of advances in our understanding of bone marrow function and architecture in humans and animals in recent years ${ }^{102}$. In parallel,

Fig. 5 | Revisiting interventions to block Plasmodium falciparum transmission. a|Current transmission-blocking vaccines target parasite processes in the mosquito, posing formidable technical challenges. Antibodies are taken up with the few microlitres of a mosquito blood meal and require high titres in the human blood. Many of the target proteins are not expressed during human infection, and hence there is no natural boosting of the immune response. Finally, efficacy testing requires mosquito feeding assays, which are cumbersome. $\mathbf{b}$ |Alternatively, transmission can be blocked by targeting gametocytes (green) with stage $\mathrm{V}$ density in the circulating blood as a readout. Many antimalarials that are active against asexual bloodstage parasites (blue) are also active against immature gametocytes, including artemisinin and its derivatives. A few antimalarials are mostly active against stage $V$ gametocytes, in particular primaquine. Identification of the bone marrow as a reservoir for asexual parasites and gametocytes opens new opportunities for interventions targeting both asexual parasite burden and transmission: (i) vaccines or human monoclonal antibodies could target receptor-ligand interactions required for parasite homing and extravasation, thereby blocking establishment of bone marrow infection and gametocyte development; (ii) vaccines or antibodies could also inhibit interactions at the erythroblast island or with mesenchymal stem cells, possibly triggering premature parasite release into the circulation and subsequent clearance in the spleen; and (iii) drugs that inhibit the deformability switch may lead to the accumulation of mature gametocytes in the bone marrow parenchyma and hence prevent their release into the circulation and their transmission. 
a series of technical breakthroughs were made, such as bone marrow-on-a-chip models for mouse and human ${ }^{103,104}$, humanized mouse models with a human bone marrow niche ${ }^{105}$, bone marrow and parasite atlases based on single-cell RNA sequencing ${ }^{106,107}$ and advances in tissue imaging, including intravital approaches ${ }^{58}$. We anticipate that these recent developments will enable future research to address some of the major questions. How does parasite infection affect bone marrow function, including immunity? What is the molecular basis for parasite phenotypes, such as bone marrow homing and extravasation of young parasites, host-parasite interactions and intravasation of maturing parasites, and mobility of mature gametocytes? Can the first wave of merozoites emerging from the liver schizont directly home to the bone marrow? What is the role of the spleen as a reservoir for asexual parasites and gametocytes in different parasite lineages? Can we develop in vitro culture systems for $P$. vivax using bone marrow proxies? Is bone marrow recrudescence an alternative relapse mechanism in $P$. vivax and other parasites with hypnozoites? Closing these knowledge and tool gaps may translate into novel diagnostics and interventions to eliminate and eventually eradicate these deadly human parasites.

Published online 9 January 2020
1. WHO. World Malaria Report 2018. https://www.who. int/malaria/publications/world-malaria-report-2018/ report/en/ (2018)

2. Tavares, J. et al. Role of host cell traversal by the malaria sporozoite during liver infection. J. Exp. Med. 210, 905-915 (2013).

3. Amino, R. et al. Quantitative imaging of Plasmodium transmission from mosquito to mammal. Nat. Med. 12 220-224 (2006).

4. Mota, M. M. et al. Migration of Plasmodium sporozoites through cells before infection. Science 291, 141-144 (2001).

5. Sturm, A. et al. Manipulation of host hepatocytes by the malaria parasite for delivery into liver sinusoids. Science 313, 1287-1290 (2006).

6. Sologub, L. et al. Malaria proteases mediate insideout egress of gametocytes from red blood cells following parasite transmission to the mosquito Cell Microbiol. 13, 897-912 (2011).

7. Janse, C. J., Ramesar, J., van den Berg, F. M. \& Mons, B. Plasmodium berghei: in vivo generation and selection of karyotype mutants and non-gametocyte producer mutants. Exp. Parasitol. 74, 1-10 (1992).

8. Alano, P. et al. Plasmodium falciparum: parasites defective in early stages of gametocytogenesis. Exp. Parasitol. 81, 227-235 (1995).

9. Kafsack, B. F. et al. A transcriptional switch underlies commitment to sexual development in malaria parasites. Nature 507, 248-252 (2014).

10. Sinha, A. et al. A cascade of DNA-binding proteins for sexual commitment and development in Plasmodium. Nature 507, 253-257 (2014).

11. Brancucci, N. M. B. et al. Heterochromatin protein 1 secures survival and transmission of malaria parasites. Cell Host Microbe 16, 165-176 (2014).

12. Coleman, B. I. et al. A Plasmodium falciparum histone deacetylase regulates antigenic variation and gametocyte conversion. Cell Host Microbe 16 177-186 (2014)

13. Eksi, S. et al. Plasmodium falciparum gametocyte development 1 (Pfgdv1) and gametocytogenesis early gene identification and commitment to sexual development. PLOS Pathog. 8, e1002964 (2012).

14. Filarsky, M. et al. GDV1 induces sexual commitment of malaria parasites by antagonizing HP1-dependent gene silencing. Science 359, 1259-1263 (2018).

15. Buckling, A., Ranford-Cartwright, L. C., Miles, A. $\&$ Read, A. F. Chloroquine increases Plasmodium falciparum gametocytogenesis in vitro. Parasitology 118 (Pt 4), 339-346 (1999).

16. Buchholz, K. et al. A high-throughput screen targeting malaria transmission stages opens new avenues for drug development. J. Infect. Dis. 203, 1445-1453 (2011).

17. Brancucci, N. M. B. et al. Lysophosphatidylcholine regulates sexual stage differentiation in the human malaria parasite Plasmodium falciparum. Cell 171, 1532-1544.e15 (2017).

18. Brancucci, N. M. B. et al. Probing Plasmodium falciparum sexual commitment at the single-cell level. Wellcome Open Res. 3, 70 (2018).

19. Poran, A. et al. Single-cell RNA sequencing reveals a signature of sexual commitment in malaria parasites. Nature 551, 95-99 (2017).

20. Brancucci, N. M., Witmer, K., Schmid, C. \& Voss, T. S. A var gene upstream element controls protein synthesis at the level of translation initiation in Plasmodium falciparum. PLOS ONE 9, e 100183 (2014).
21. Josling, G. A. \& Llinas, M. Sexual development in Plasmodium parasites: knowing when it's time to commit. Nat. Rev. Microbiol. 13, 573-587 (2015).

22. Josling, G. A., Williamson, K. C. \& Llinas, M. Regulation of sexual commitment and gametocytogenesis in malaria parasites. Annu. Rev. Microbiol. 72, 501-519 (2018).

23. Hawking, F., Wilson, M. E. \& Gammage, K. Evidence for cyclic development and short-lived maturity in the gametocytes of Plasmodium falciparum. Trans. R. Soc Trop. Med. Hyg. 65, 549-559 (1971).

24. Gautret, P. \& Motard, A. Periodic infectivity of Plasmodium gametocytes to the vector. A review. Parasite 6, 103-111 (1999)

25. Dearnley, M. et al. Reversible host cell remodeling underpins deformability changes in malaria parasite sexual blood stages. Proc. Natl Acad. Sci. USA 113, 4800-4805 (2016).

26. Hliscs, M. et al. Organization and function of an actin cytoskeleton in Plasmodium falciparum gametocytes. Cell. Microbiol. 17, 207-225 (2015).

27. Dearnley, M. K. et al. Origin, composition, organization and function of the inner membrane complex of Plasmodium falciparum gametocytes. J. Cell Sci. 125, 2053-2063 (2012).

28. Turner, L. et al. Severe malaria is associated with parasite binding to endothelial protein $\mathrm{C}$ receptor Nature 498, 502-505 (2013).

29. Seydel, K. B. et al. Brain swelling and death in children with cerebral malaria. N. Engl. J. Med. 372, 1126-1137 (2015).

30. Fried, M. \& Duffy, P. E. Adherence of Plasmodium falciparum to chondroitin sulfate a in the human placenta. Science 272, 1502-1504 (1996).

31. Cranston, H. A. et al. Plasmodium falciparum maturation abolishes physiologic red cell deformability. Science 223, 400-403 (1984).

32. Ginsburg, H. \& Krugliak, M. Uptake of L-tryptophan by erythrocytes infected with malaria parasites (Plasmodium falciparum). Biochim. Biophys. Acta 729, 97-103 (1983)

33. Vestweber, D. How leukocytes cross the vascular endothelium. Nat. Rev. Immunol. 15, 692-704 (2015).

34. Leech, J. H., Barnwell, J. W., Miller, L. H. \& Howard, R. J. Identification of a strain-specific malarial antigen exposed on the surface of Plasmodium falciparum-infected erythrocytes. J. Exp. Med. 159 1567-1575 (1984).

35. Su, X. Z. et al. The large diverse gene family var encodes proteins involved in cytoadherence and antigenic variation of Plasmodium falciparum-infected erythrocytes. Cell 82, 89-100 (1995)

36. Smith, J. D. et al. Identification of a Plasmodium falciparum intercellular adhesion molecule- 1 binding domain: a parasite adhesion trait implicated in cerebral malaria. Proc. Natl Acad. Sci. USA 97 , 1766-1771 (2000).

37. Salanti, A. et al. Evidence for the involvement of VAR2CSA in pregnancy-associated malaria. J. Exp. Med. 200, 1197-1203 (2004).

38. Flick, K. et al. Role of nonimmune IgG bound to PfEMP1 in placental malaria. Science 293, 2098-2100 (2001).

39. Chan, J. A. et al. Targets of antibodies against Plasmodium falciparum-infected erythrocytes in malaria immunity. J. Clin. Invest. 122, 3227-3238 (2012).

40. Kyes, S. A., Rowe, J. A., Kriek, N. \& Newbold, C. I. Rifins: a second family of clonally variant proteins expressed on the surface of red cells infected with Plasmodium falciparum. Proc. Natl Acad. Sci. USA 96, 9333-9338 (1999).

41. Cheng, Q. et al. Stevor and rif are Plasmodium falciparum multicopy gene families which potentially encode variant antigens. Mol. Biochem. Parasitol. 97, 161-176 (1998)

42. Niang, M. et al. STEVOR is a Plasmodium falciparum erythrocyte binding protein that mediates merozoite invasion and rosetting. Cell Host Microbe 16, 81-93 (2014).

43. Goel, S. et al. RIFINs are adhesins implicated in severe Plasmodium falciparum malaria. Nat. Med. 21, 314-317 (2015).

44. Smith, C. D., Brown, A. E., Nakazawa, S., Fujioka, H. \& Aikawa, M. Multi-organ erythrocyte sequestration and ligand expression in rhesus monkeys infected with Plasmodium coatneyi malaria. Am. J. Trop. Med. Hyg. 55, 379-383 (1996)

45. Handayani, S et al. High deformability of Plasmodium vivax-infected red blood cells under microfluidic conditions. J. Infect. Dis. 199, 445-450 (2009).

46. Marchiafava, E. \& Bignami, A. Sulle febbri estivo aumnali (E. Loescher, 1894).

47. Baro, B. et al. Plasmodium vivax gametocytes in the bone marrow of an acute malaria patient and changes in the erythroid miRNA profile. PLOS Negl. Trop. Dis. 11, e0005365 (2017)

48. Farfour, E., Charlotte, F., Settegrana, C., Miyara, M $\&$ Buffet, P. The extravascular compartment of the bone marrow: a niche for Plasmodium falciparum gametocyte maturation? Malar. J. 11, 285 (2012)

49. Smalley, M. E., Abdalla, S. \& Brown, J. The distribution of Plasmodium falciparum in the peripheral blood and bone marrow of Gambian children. Trans. R. Soc. Trop. Med. Hyg. 75 103-105 (1981).

50. Thomson, J. G. \& Robertson, A. The structure and development of Plasmodium falciparum gametocytes in the internal organs and peripheral circulation. Trans. R. Soc. Trop. Med. Hyg. 14, 31-40 (1935).

51. Joice, R. et al. Plasmodium falciparum transmission stages accumulate in the human bone marrow. Sci. Transl. Med. 6, 244re245 (2014).

This study provides quantitative evidence for $P$. falciparum gametocyte enrichment in the human bone marrow.

52. Aguilar, R. et al. Molecular evidence for the localization of Plasmodium falciparum immature gametocytes in bone marrow. Blood 123, 959-966 (2014).

53. Ellis, R. E. The distribution of active bone marrow in the adult. Phys. Med. Biol. 5, 255-258 (1961).

54. Woodard, H. Q. \& Holodny, E. A summary of the data of Mechanik on the distribution of human bone marrow. Phys. Med. Biol. 5, 57-59 (1960).

55. Nombela-Arrieta, C. \& Manz, M. G. Quantification and three-dimensional microanatomical organization of the bone marrow. Blood Adv. 1, 407-416 (2017).

56. Chasis, J. A. \& Mohandas, N. Erythroblastic islands: niches for erythropoiesis. Blood 112, 470-478 (2008).

57. Obaldia, N., 3rd et al. Bone marrow is a major parasite reservoir in Plasmodium vivax infection. MBio 8, 1-16 (2018).

This study provides quantitative evidence for $P$. vivax gametocyte enrichment and an asexual reservoir in the bone marrow of non-human primates. 
58. De Niz, M. et al. Plasmodium gametocytes display homing and vascular transmigration in the host bone marrow. Sci. Adv. 4, eaat3775 (2018). This study shows a $P$. berghei asexual and gametocyte reservoir in the bone marrow and spleen of infected mice and provides the first description of parasite transmigration events across the endothelial barrier.

59. Duffier, Y. et al. A humanized mouse model for sequestration of Plasmodium falciparum sexual stages and in vivo evaluation of gametocytidal drugs. Sci. Rep. 6, 35025 (2016).

This study demonstrates $P$. faciparum gametocyte enrichment in the bone marrow and spleen of humanized mice

60. Lee, R. S., Waters, A. P. \& Brewer, J. M. A cryptic cycle in haematopoietic niches promotes initiation of malaria transmission and evasion of chemotherapy. Nat. Commun. 9, 1689 (2018)

This study provides evidence that an asexual $P$. berghei parasite reservoir in the spleen of infected mice can lead to recrudescence.

61. Lopes, S. C. et al. Paucity of Plasmodium vivax mature schizonts in peripheral blood is associated with their increased cytoadhesive potential. J. Infect. Dis. 209 1403-1407 (2014).

62. Lim, C. et al. Reticulocyte preference and stage development of Plasmodium vivax Isolates. J. Infect Dis. 214, 1081-1084 (2016).

63. Fonseca, L. L., Joyner, C. J., Ma, H. C., Galinski, M. R. \& Voit, E. O. A model of Plasmodium vivax concealment based on Plasmodium cynomolgi infections in Macaca mulatta. Malar. J. 16, 375 (2017)

64. Usui, M. et al. Plasmodium falciparum sexual differentiation in malaria patients is associated with host factors and GDV1-dependent genes. Nat. Commun 10, 2140 (2019).

65. Farid, R., Dixon, M. W., Tilley, L. \& McCarthy, J. S Initiation of gametocytogenesis at very low parasite density in Plasmodium falciparum infection. J. Infect. Dis. 215, 1167-1174 (2017).

66. Rono, M. K. et al. Adaptation of Plasmodium falciparum to its transmission environment. Nat. Ecol. Evol. 2, 377-387 (2018).

67. Pelle, K. G. et al. Transcriptional profiling defines dynamics of parasite tissue sequestration during malaria infection. Genome Med. 7, 19 (2015).

68. Perrin, A. J., Bartholdson, S. J. \& Wright, G. J. P-selectin is a host receptor for Plasmodium MSP7 ligands. Malar. J. 14, 238 (2015)

69. Avril, M., Brazier, A. J., Melcher, M., Sampath, S. \& Smith, J. D. DC8 and DC13 var genes associated with severe malaria bind avidly to diverse endothelial cells. PLOS Pathog. 9, e1003430 (2013).

70. Rogers, N. J., Hall, B. S., Obiero, J., Targett, G. A. \& Sutherland, C. J. A model for sequestration of the transmission stages of Plasmodium falciparum: adhesion of gametocyte-infected erythrocytes to human bone marrow cells. Infect. Immun. $\mathbf{6 8}$ 3455-3462 (2000)

71. Tiburcio, M. et al. Early gametocytes of the malaria parasite Plasmodium falciparum specifically remodel the adhesive properties of infected erythrocyte surface. Cell. Microb. 15, 647-659 (2012).

72. Silvestrini, F., Tiburcio, M., Bertuccini, L. \& Alano, P. Differential adhesive properties of sequestered asexual and sexual stages of Plasmodium falciparum on human endothelial cells are tissue independent. PLOS ONE 7, e31567 (2012).

73. Fraschka, S. A. et al. Comparative heterochromatin profiling reveals conserved and unique epigenome signatures linked to adaptation and development of malaria parasites. Cell Host Microbe 23, 407-420.e8 (2018).

74. Messina, V. et al. Gametocytes of the malaria parasite Plasmodium falciparum interact with and stimulate bone marrow mesenchymal cells to secrete angiogenetic factors. Front. Cell. Infect. Microbiol. 8, 50 (2018). This study provides evidence for binding of asexual and gametocyte iRBCs to bone marrow MSCs.

75. Neveu, G. et al. Plasmodium falciparum gametocyteinfected erythrocytes do not adhere to human primary erythroblasts. Sci. Rep. 8, 17886 (2018).

76. Silvestrini, F. et al. Protein export marks the early phase of gametocytogenesis of the human malaria parasite Plasmodium falciparum. Mol. Cell. Proteomics 9, 1437-1448 (2010)

77. Dantzler, K. W. et al. Naturally acquired immunity against immature Plasmodium falciparum gametocytes. Sci. Transl. Med. 11, 1-14 (2019). This study identifies shared antigens on the asexual and gametocyte iRBC surface.
78. Hermand, P. et al. Plasmodium falciparum proteins involved in cytoadherence of infected erythrocytes to chemokine CX3CL1. Sci. Rep. 6, 33786 (2016).

79. Honczarenko, M. et al. Human bone marrow stromal cells express a distinct set of biologically functional chemokine receptors. Stem Cell 24, 1030-1041 (2006).

80. Tamez, P. A., Liu, H., Fernandez-Pol, S., Haldar, K. \& Wickrema, A. Stage-specific susceptibility of human erythroblasts to Plasmodium falciparum malaria infection. Blood 114, 3652-3655 (2009).

81. Tiburcio, M. et al. A switch in infected erythrocyte deformability at the maturation and blood circulation of Plasmodium falciparum transmission stages. Blood 119, e172-e180 (2012).

82. Aingaran, M. et al. Host cell deformability is linked to transmission in the human malaria parasite Plasmodium falciparum. Cell. Microbiol. 14, 983-993 (2012).

83. Waugh, R. E. Reticulocyte rigidity and passage through endothelial-like pores. Blood 78, 3037-3042 (1991).

84. Ramdani, G. et al. cAMP-signalling regulates gametocyte-infected erythrocyte deformability required for malaria parasite transmission. PLOS Pathog. 11, e1004815 (2015).

This study demonstrates that gametocyte deformability can be targeted with drugs.

85. Naissant, B. et al. Plasmodium falciparum STEVOR phosphorylation regulates host erythrocyte deformability enabling malaria parasite transmission. Blood 127, e42-e53 (2016).

86. Nixon C. P. Plasmodium falciparum gametocyte transit through the cutaneous microvasculature: a new target for malaria transmission blocking vaccines? Hum. Vaccin. Immunother 12, 3189-3195 (2016).

87. Lawniczak, M. K. \& Eckhoff, P. A. A computational lens for sexual-stage transmission, reproduction, fitness and kinetics in Plasmodium falciparum. Malar. J. 15, 487 (2016).

88. Srivastava, A. et al. Host reticulocytes provide metabolic reservoirs that can be exploited by malaria parasites. PLOS Pathog. 11, e1004882 (2015).

89. Mu, C. F. et al. Targeted drug delivery for tumor therapy inside the bone marrow. Biomaterials 155, 191-202 (2018).

90. Ng, C. L. \& Fidock, D. A. Plasmodium falciparum in vitro drug resistance selections and gene editing. Methods Mol. Biol. 2013, 123-140 (2019).

91. Camarda, G. et al. Antimalarial activity of primaquine operates via a two-step biochemical relay. Nat. Commun 10, $3226(2019)$

92. Duez, J. et al. High-throughput microsphiltration to assess red blood cell deformability and screen for malaria transmission-blocking drugs. Nat. Protoc. 13, 1362-1376 (2018)

93. Lavazec, C. \& Naissant, B. Viagra ${ }^{\circledR}$ makes Plasmodium stiff: a novel way to block malaria transmission? [French]. Med. Sci. 31, 826-828 (2015).

94. Nemazee, D. Mechanisms of central tolerance for B cells. Nat. Rev. Immunol. 17, 281-294 (2017)

95. Vainieri, M. L. et al. Systematic tracking of altered haematopoiesis during sporozoite-mediated malaria development reveals multiple response points. Open Biol. 6, 1-13 (2016).

96. Bockstal, V., Geurts, N. \& Magez, S. Acute disruption of bone marrow $B$ lymphopoiesis and apoptosis of transitional and marginal zone $B$ cells in the spleen following a blood-stage Plasmodium chabaudi infection in mice. J. Parasitol. Res. 2011, 534697 (2011).

97. Nduati, E. W. et al. Distinct kinetics of memory B-cell and plasma-cell responses in peripheral blood following a blood-stage Plasmodium chabaudi infection in mice. PLOS ONE 5, e15007 (2010).

98. Markus, M. B. New evidence for hypnozoiteindependent Plasmodium vivax malarial recurrences. Trends Parasitol. 34, 1015-1016 (2018).

99. Nothelfer, K., Sansonetti, P. J. \& Phalipon, A. Pathogen manipulation of $B$ cells: the best defence is a good offence. Nat. Rev. Microbiol. 13, 173-184 (2015).

100. Das, B. et al. CD271+ bone marrow mesenchymal stem cells may provide a niche for dormant Mycobacterium tuberculosis. Sci. Transl. Med. 5, 170ra113 (2013).

101. Varma, N. \& Naseem, S. Hematologic changes in visceral leishmaniasis/kala azar. Indian J. Hematol. Blood Transfus. 26, 78-82 (2010).

102. Pinho, S. \& Frenette, P. S. Haematopoietic stem cell activity and interactions with the niche. Nat. Rev. Mol. Cell Biol. 20, 303-320 (2019).

103. Torisawa, Y. S. et al. Bone marrow-on-a-chip replicates hematopoietic niche physiology in vitro. Nat. Meth. 11 663-669 (2014).
104. Sieber, S. et al. Bone marrow-on-a-chip: long-term culture of human haematopoietic stem cells in a three-dimensional microfluidic environment. J. Tissue Eng. Regen. Med. 12, 479-489 (2018) This study describes the first human bone marrow-on-a-chip platform

105. Rahmig, S. et al. Improved human erythropoiesis and platelet formation in humanized NSGW41 mice. Stem Cell Rep. 7, 591-601 (2016)

106. Baryawno, N. et al. A cellular taxonomy of the bone marrow stroma in homeostasis and leukemia. Cell 177, 1915-1932.e16 (2019). This study provides a single-cell atlas of the human bone marrow niche.

107. Howick, V. M. et al. The Malaria Cell Atlas: single parasite transcriptomes across the complete Plasmodium life cycle. Science 365, 1-11 (2019). This study provides a single-cell atlas of the $P$. falciparum life cycle.

108. Telford, S. R. in The Hemoparasites of the Reptilia: Color Atlas and Text 376 pp. (CRC, 2009).

109. Garnham, P. C. C. in Malaria Parasites and Other Haemosporidia 1132 pp. (Blackwell, 1966).

110. Valkiunas, G. in Avian Malaria Parasites and Other Haemosporidia 946 pp. (CRC Press, 2005).

111. Palinauskas, V. et al. Description, molecular characterisation, diagnostics and life cycle of Plasmodium elongatum (lineage pERIRUB01), the virulent avian malaria parasite. Int. J. Parasitol. 46 697-707 (2016)

This work is one of several avian malaria studies describing erythrocytic schizonts in the bone marrow of infected birds.

112. Perkins, S. L. Malaria's many mates: past, present, and future of the systematics of the order Haemosporida. J. Parasitol. 100, 11-25 (2014)

113. MacCallum, W. G. On the flagellated form of the malaria parasite. Lancet 11, 1240-1241 (1897)

114. Aragão, H. B. Über den Entwicklungsgang and die Übertragung von Haemoproteus columbae. Arch. Protistenkunde 12, 154-167 (1908).

115. James, S. P. \& Tate, P. New knowledge of the life-cycle of malaria parasites. Nature 139, 545 (1937).

116. Ross, R. Report on the cultivation of Proteosoma Labbé, in grey mosquitoes. Indian Med. Gaz. 33 , 401-408 (1898).

117. Coatney, G. R., Cooper, W. C., Eddy, N. B. \& Greenberg, J. Survey of antimalarial agents: chemotherapy of Plasmodium gallinaceum infections; toxicity; correlation of structure and action. Public Health Monogr. 9, 1-322 (1953).

118. Hawking, F. Tissue culture of malaria parasites (Plasmodium gallinaceum). Lancet 246, 693-694 (1944).

119. Ilgunas, M. et al. Patterns of Plasmodium homocircumflexum virulence in experimentally infected passerine birds. Malar. J. 18, 174 (2019).

120. Valkiunas, G. \& lezhova, T. A. Exo-erythrocytic development of avian malaria and related haemosporidian parasites. Malar. J. 16, 101 (2017). This review is the first on exo-erythrocytic development of avian malaria parasites, including cells of the haematopoietic niche.

121. Ben-Harel, S. Studies of bird malaria in relation to the mechanism of relapse. Am. J. Hyg. 3, 652 (1923).

122. Ilgunas, M., Palinauskas, V., Platonova, E., lezhova, T. $\&$ Valkiunas, G. The experimental study on susceptibility of common European songbirds to Plasmodium elongatum (lineage pGRW6), a widespread avian malaria parasite. Malar. J. 18, 290 (2019). This study provides evidence that virulence is related to damage of bone marrow cells during avian malaria.

123. Lainson, R. in Atlas of Protozoan Parasites of the Amazonian fauna of Brazil. Volume 1: Haemosporidia of Reptiles 81 pp. (Instituto Evandro Chagas, 2012).

124. Schaer, J. et al. Nycteria parasites of Afrotropical insectivorous bats. Int. J. Parasitol. 45, 375-384 (2015).

125. Schaer, J. et al. Epauletted fruit bats display exceptionally high infections with a Hepatocystis species complex in South Sudan. Sci. Rep. 7, 6928 (2017).

126. Galen, S. C. et al. The polyphyly of Plasmodium: comprehensive phylogenetic analyses of the malaria parasites (order Haemosporida) reveal widespread taxonomic conflict. R. Soc. Open. Sci. 5, 171780 (2018). This study presents a comprehensive phylogenetic analysis of Haemosporidia, proposing the polyphyletic status of the genus Plasmodium. 
127. Borner, J. et al. Phylogeny of haemosporidian blood parasites revealed by a multi-gene approach. Mol. Phylogenet. Evol. 94, 221-231 (2016).

128. Galen, S. C., Nunes, R., Sweet, P. R. \& Perkins, S. L. Integrating coalescent species delimitation with analysis of host specificity reveals extensive cryptic diversity despite minimal mitochondrial divergence in the malaria parasite genus Leucocytozoon. BMC Evol. Biol. 18, 128 (2018).

\section{Acknowledgements}

The authors thank Chris Moxon (University of Glasgow) Juliane Schär (Humboldt University, Berlin) and members of the Marti laboratory for critical reading of the manuscript and helpful discussions. Research in the Marti laboratory is supported by a Wellcome Senior Investigator award (M.M.) European Research Council Consolidator award BoneMalar (M.M.) and a Wellcome Trust Centre award to the Wellcome Center for Integrative Parasitology. Additiona funding comes from a Royal Society Wolfson Merit award (M.M.) and a German Research Foundation postdoctora fellowship (F.H.)

Author contributions

All authors researched data for the article, wrote the article and reviewed or edited the manuscript before submission.
Competing interests

The authors declare no competing interests.

\section{Peer review information}

Nature Reviews Microbiology thanks Pietro Alano, Tania de Koning-Ward and the other, anonymous, reviewer(s) for their contribution to the peer review of this work.

\section{Publisher's note}

Springer Nature remains neutral with regard to jurisdictional claims in published maps and institutional affiliations.

(c) Springer Nature Limited 2020 